\title{
Simulación del comportamiento elastoplástico de estructuras bifásicas policristalinas de aceros "Dual-phase".
}

\author{
E.A. BONIFAZ, A. MARTÍN MEIZOSO, J.M. MARTÍNEZ ESNAOLA, J. GIL SEVILLANO \\ Centro de Estudios e Investigaciones Técnicas de Gipuzkoa (CEIT) y Escuela Superior de Ingenieros de San Sebastián (Universidad de Navarra); \\ Paseo de Manuel Lardizábal 15, 20018 San Sebastián
}

\begin{abstract}
Mediante el Método de los Elementos Finitos (MEF) se han realizado simulaciones del comportamiento elastoplástico de estructuras bifásicas policristalinas con un mismo comportamiento elástico y diferente comportamiento plástico, representativas de aceros bifásicos tipo "dual-phase" (ferrita/martensita). Las simulaciones se realizan en 3 dimensiones, con diferentes condiciones de contorno: caras planas, y caras libres. Se analiza el efecto de la morfología y del número de los granos de ambas fases contenidos en la muestra analizada sobre la curva macroscópica tensión-deformación y sobre varios aspectos micromecánicos de la predicción, tales como la distribución de las deformaciones en las dos fases, la heterogeneidad de las deformaciones locales o las tensiones residuales a escala mesoscópica. Los resultados orientan sobre los métodos más eficientes para la simulación a diferentes escalas del comportamiento plástico de materiales bifásicos y pueden servir de ayuda para el diseño microestructural de nuevos materiales.
\end{abstract}

Palabras clave: Elementos finitos, aceros "dual-phase", comportamiento elastoplástico, materiales bifásicos.

\section{Simulation of elasto-plastic behaviour of polycristalline dual-phase steel microestructures}

In order to model the elasto-plastic behaviour of polycrystalline dual-phase steel microstructures (ferrite/martensite), uniaxial tension is simulated using a 3-D finite element code. Calculations are performed for a representative dual-phase cubic volume discretised in 3375 elements, using either plane or free-face boundary conditions. The effects of morphology and grain number on the stress-strain macroscopic curve and on the strain distribution are analised. The results confirm the efficiency of the method for predicting the elasto-plastic behaviour of dual-phase steels. The simulations are aimed to be used for the microstructural design of new dual-phase steels.

Keywords: Finite element method, dual-phase steels, elasto-plastic behaviour, two-phase materials.

\section{INTRODUCCIÓN}

Los aceros bifásicos "dual-phase" constituidos por ferrita/ martensita combinan alta resistencia y excelente conformabilidad (1-3). Se obtienen por una transformación intercrítica y enfriamiento rápido desde la región bifásica ferrita/austenita. El control de la estructura de partida, del recocido intercrítico y del enfriamiento posterior permite conseguir una gama amplísima de proporciones de los dos constituyentes y de su topología. La predicción del comportamiento mecánico de tales aceros, conocido el comportamiento individual de las fases componentes, es una tarea que involucra estudios teóricos, experimentales y numéricos. Actualmente, el problema continúa sin resolverse en su totalidad; esto se debe principalmente al reparto irregular de tensiones y deformaciones que se producen en materiales bifásicos policristalinos, ocasionado por las complejas relaciones geométricas (número de granos, morfología) y diferente distribución espacial de las fases. Este trabajo, que utiliza el Método de los Elementos Finitos (MEF), permite apreciar cómo la distribución de ambas fases, su fracción volumétrica y su morfología así como las condiciones de contorno seleccionadas, influyen en la curva macroscópica tensióndeformación del acero y en la distribución de deformaciones plásticas y tensiones residuales en su interior.

\section{HIPÓTESIS DEL MODELO}

Se simula, mediante elementos finitos, la deformación a tracción de un volumen representativo del material bifásico subdi- vidido en elementos cúbicos. La fracción volumétrica de cada fase se considera al designar elementos de fase 1 o de fase 2 mediante el generador de microestructuras virtuales RANVORJM $(4,5)$. Para los cálculos, se establecen las condiciones de simetría en tres caras ortogonales (Fig. 1), y se impone un desplazamiento uniforme sobre la cara perpendicular a la dirección de la tracción (dirección 2). Las otras dos caras son forzadas a permanecer planas y paralelas a sí mismas (caras planas), o son libres de deformarse (caras libres).

Los cálculos se han realizado con el paquete de elementos finitos ABAQUS (6) utilizando elementos C3D8 (elementos sólidos -“bricks"- cúbicos de 8 nudos). El comportamiento mecánico de la fase dura (martensita) se supone elástico-plástico perfecto, con un valor de tensión de fluencia constante $\sigma_{y}=$ $2000 \mathrm{MPa}$, mientras que la fase blanda (ferrita) presenta un endurecimiento lineal dado por $\sigma_{y}=300+1000 \varepsilon(\mathrm{MPa})$. Las constantes elásticas utilizadas son idénticas para las dos fases: $E=200$ GPa y $v=0.3$ ( $E$ es el módulo de Young, $v$ es el coeficiente de Poisson). Para la obtención de la curva macroscópica tensión-deformación ( $\sigma$ vs. $\varepsilon$ ), la tensión verdadera $\sigma$ se deduce del promedio de las tensiones normales que se producen en la cara donde se aplica el desplazamiento $(7,8)$;

$$
\sigma=\frac{1}{A} \int_{A} \sigma_{22} d A
$$

La deformación verdadera se obtiene a partir del desplaza- 
miento impuesto en la dirección 2;

$$
\varepsilon=\ln \left(\frac{l_{0}+\mu_{2}}{l_{o}}\right)
$$

siendo $1_{\mathrm{o}}$ la arista inicial del cubo.

\section{RESULTADOS Y DISCUSIÓN}

Se presentan resultados correspondientes a un bifásico con $50 \%$ de ambas fases, discretizando el volumen en 15 x 15 x15 elementos monofásicos.

En la Fig. 2 se aprecia el efecto de las condiciones de contorno calculado en un volumen representativo de material bifásico compuesto de 12 granos (cada grano se halla compuesto como promedio por 281 elementos C3D8) generados aleatoriamente teselando el volumen de acuerdo con una ley de Voronoi (5). Los valores más altos corresponden a una situación de caras planas, más restrictiva que la situación que permite desplazamientos fuera del plano en esas caras (creación de rugosidad en una superficie libre). Puede estimarse que la situación real de un policristal bifásico de grano fino será intermedia entre ambas hipótesis.

La Fig. 3 muestra las curvas tensión deformación para tres distribuciones con $50 \%$ de fase dura y distinto grado de refinamiento. Se observa que la distribución aleatoria ("random cubes"), donde cada elemento cúbico actúa como un grano independiente, produce valores más altos que las otras dos, que simulan mejor la morfología bifásica.

La Fig. 4(a) muestra una discretización del volumen en 50 granos repartidos al $50 \%$ en las dos fases (las fronteras entre granos de la misma fase no se señalan). La Fig. 4(b) muestra los contornos de deformación plástica equivalente tras un alargamiento total de 0.10 . Se observa que la heterogeneidad local de deformaciones equivalentes dentro de cada fase es muy fuerte. Las Figuras 4(c) y 4(d) muestran, respectivamente, la función de distribución acumulada y el nivel de tensiones residuales $\sigma_{22}$ (2 es la dirección previa de tracción) tras la descarga. Se comprueba que la fase dura se encuentra sometida mayoritariamente a tracción y la fase blanda a compresión, y que los niveles de tensiones residuales anticipados son muy importantes, (la tensión promedio desde la que se descargó era $\left.\sigma_{(\varepsilon=0.10)}=1066.5 \mathrm{Mpa}\right)$.

\section{CONCLUSIONES}

La simulación del comportamiento elastoplástico de materiales bifásicos por medio del método de los elementos finitos es muy sensible al tipo de condiciones de contorno, distribución espacial de las fases y finura de la malla utilizada.

El acoplamiento del programa de generación de estructuras virtuales "RANVORJM" con el paquete ABAQUS ha permitido la simulación 3D del comportamiento elastoplástico de estructuras bifásicas policristalinas verosímiles.

Los resultados orientan sobre los métodos más eficientes para la simulación del comportamiento

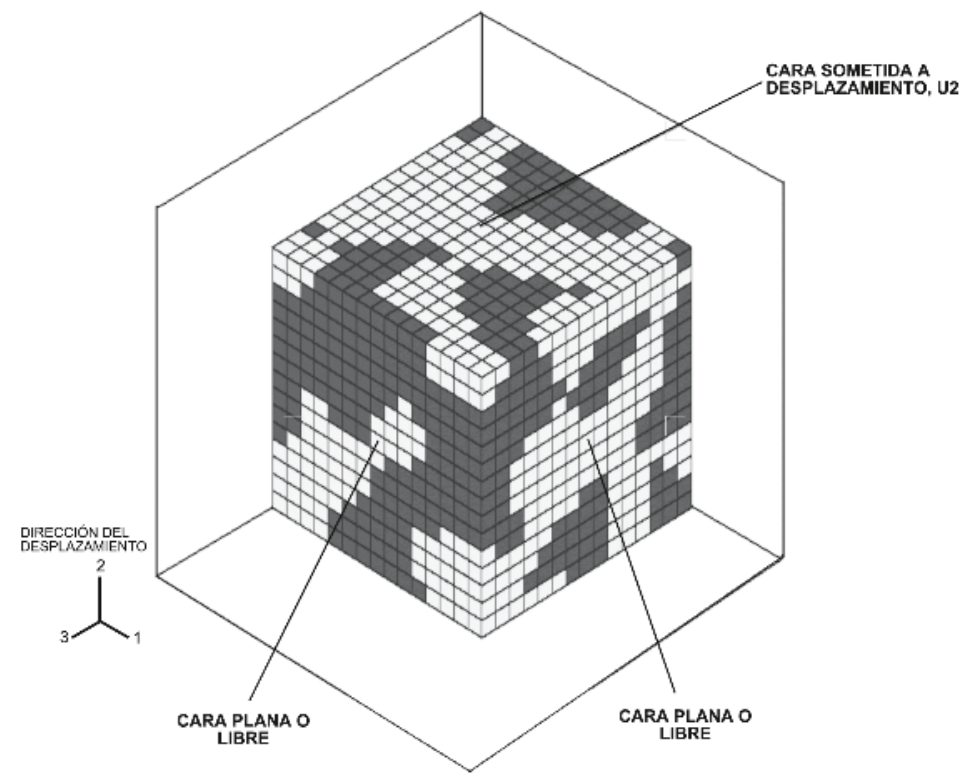

Figura 1. Representación esquemática de las condiciones de contorno empleadas en las simulaciones.

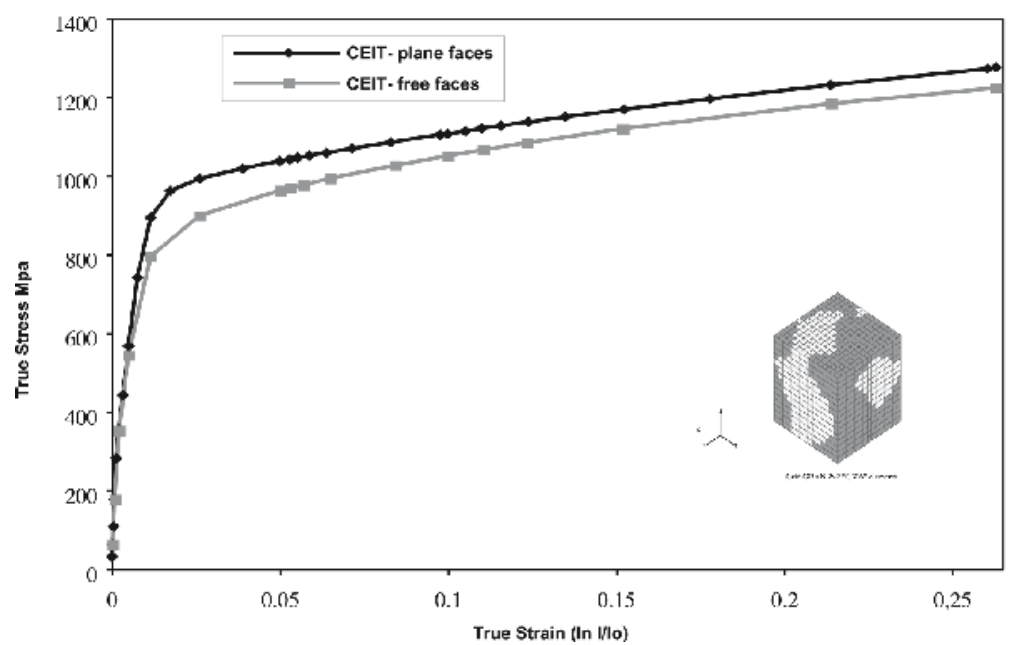

Figura 2. Efecto de las condiciones de contorno sobre un mismo tipo de distribución y malla, 12 granos, 50\% de fase dura.

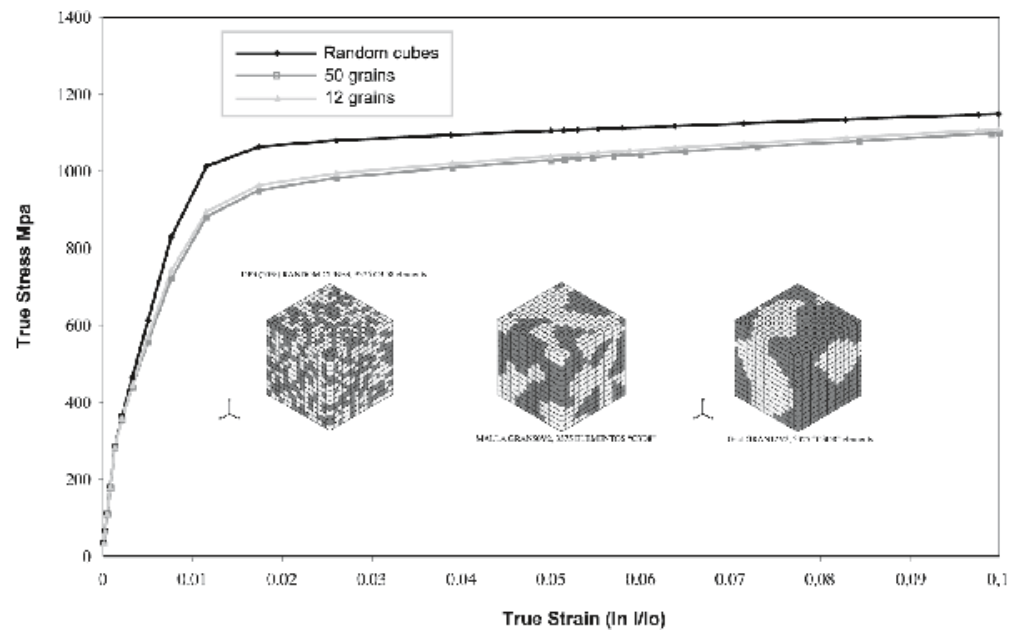

Figura 3. Curvas tensión-deformación para las tres distribuciones mostradas de fase dura (50\%). Condiciones de contorno: caras planas. 


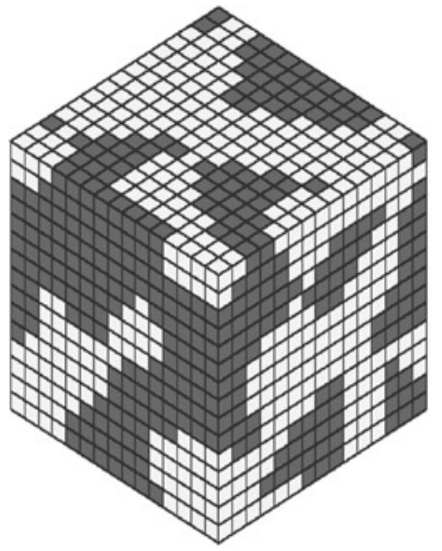

a

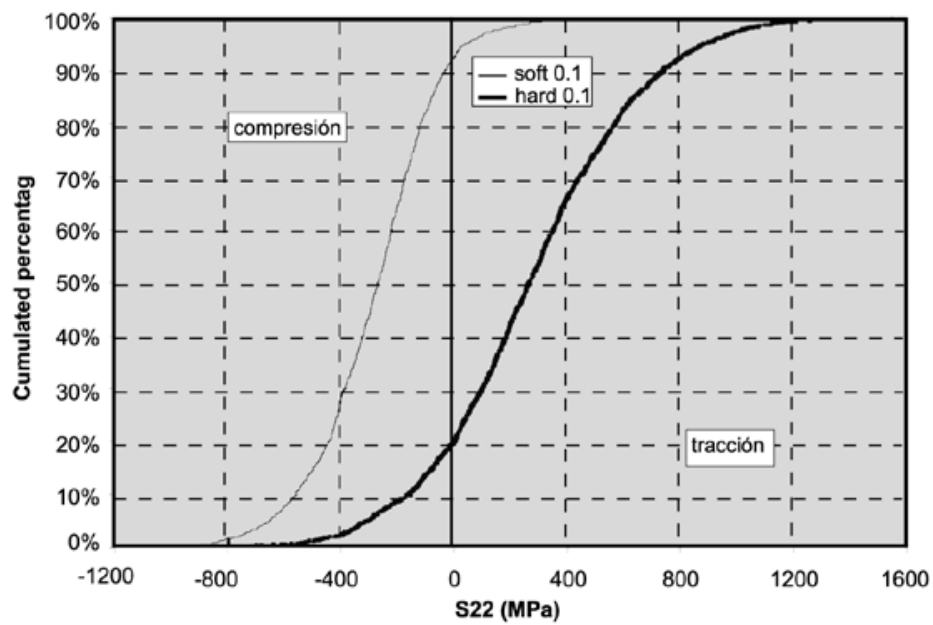

C
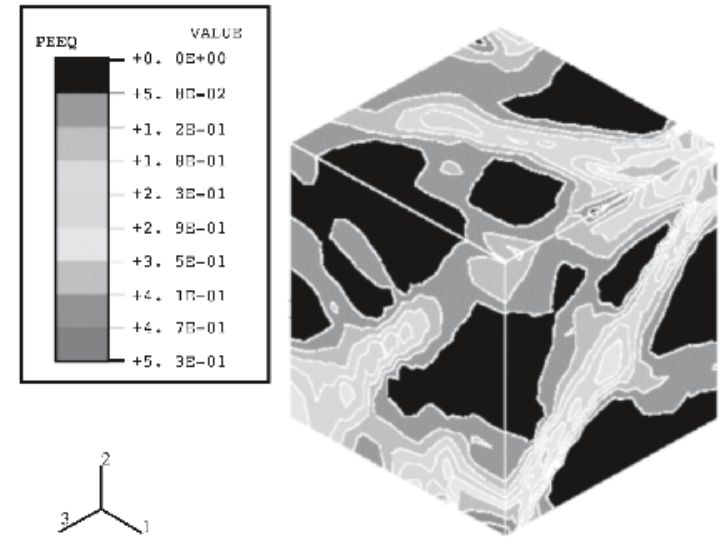

b
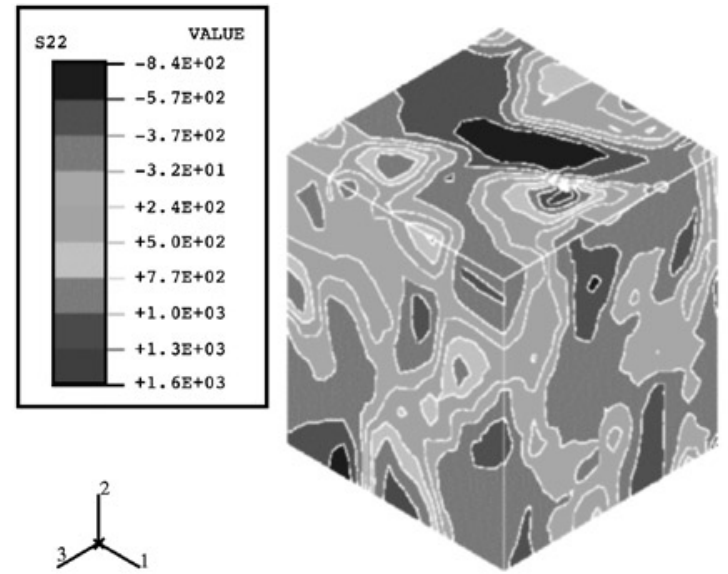

d

Figura 4. Estados de tensión y deformación para una deformación ingenieril del 10\% (s = 1066.5 Mpa) y condiciones de contorno caras planas: (a) malla representativa de una morfología de 50 granos; (b) contornos de deformación equivalente; (c) distribución acumulada de las tensiones residuales s22; (d) contornos de tensiones residuales (s22).

plástico a diferentes escalas de materiales bifásicos desde un punto de vista computacional, y sirven de ayuda para el diseño microestructural de nuevos materiales. Pueden compararse rápidamente los efectos microestructurales de la fracción en volumen, conectividad de las fases, etc., sobre la resistencia y el endurecimiento de los aceros "dual-phase". Esto posibilita la selección de estructuras optimizadas, economizando mucho trabajo experimental.

\section{AGRADECIMIENTOS}

Los resultados incluidos en este trabajo se han obtenido en el desarrollo del proyecto CECA-Acero 7210/PR/044 (European Commission, DG XII), en colaboración con IRSID (Francia), IEHK (Alemania) y Hoogovens (Holanda). E. A. Bonifaz agradece a la Agencia Española de Cooperación Internacional AECI la concesión de una beca MUTIS de doctorado, y, a la Escuela Politécnica de Chimborazo de Ecuador el continuo apoyo brindado.

\section{BIBLIOGRAFIA}

1. Balliger N.K and Gladman T., “Work hardening of dual-phase steels". Metal Science, 95-108, 1981

2. Llewellyn D.T. and Hills D.J., "Dual phase steels". Ironmaking and Steelmaking, Vol. $23 \mathrm{~N}_{0}$. 6, 471-478, 1996.

3. Gladman T., "The physical metallurgy of microalloyed steel”, IOM, 1997.

4Programa RANVORJM, Centro de Estudios e Investigaciones Técnicas de Gipuzkoa (CEIT), 1998.

5. Martín Meizoso, A., Tellaeche Repáraz, M. y Gil Sevillano, J. “Un generador aleatorio de microestructuras virtuales 3D". Revista de Metalurgia, Vol. 34, 314-318, 1998.

6. ABAQUS User's Manual 5.8, Hibbit, Karlsson and Sorensen, 1998

7. Lung, T., Buesler, P., Kaluza, W., Vrieze, J., Gil Sevillano, J., “Modelling of mechanical properties and local deformation of high strength multi-phase steels", ECSC report, 1999.

8. Leggoe, J.W., Mammoli, A.A., Bush, M.B. and Hu, X.Z., “Finite element modelling of deformation in particulate reinforced metal matrix composites with random local microstructure variation". Acta Mater., Vol. 46, No. 17, 60756088, 1998. 